\title{
Interdialytic Weight Gain in Hemodialysis Patients: Worse Hospital Admissions and Intradialytic Hypotension
}

\author{
Yaqoob Al Maimani1, Fady Elias ${ }^{1}$, Issa Al Salmi ${ }^{1,2 *}{ }^{\circledR}$, Abdullah Aboshakra1, \\ Mohamed Awad Alla1, Suad Hannawi ${ }^{3}$ (i)
}

${ }^{1}$ The Renal Medicine Department, The Royal Hospital, Muscat, Oman

${ }^{2}$ Oman Medical Specialty Board, Azzibah, Muscat, Oman

${ }^{3}$ The Medicine Department, MOHAP, Dubai, UAE

Email: *isa@ausdoctors.net

How to cite this paper: Al Maimani, Y., Elias, F., Al Salmi, I., Aboshakra, A., Alla, M.A. and Hannawi, S. (2021) Interdialytic Weight Gain in Hemodialysis Patients: Worse Hospital Admissions and Intradialytic Hypotension. Open Journal of Nephrology, 11, 156-170.

https://doi.org/10.4236/ojneph.2021.112013

Received: February 26, 2021

Accepted: April 24, 2021

Published: April 27, 2021

Copyright $\odot 2021$ by author(s) and Scientific Research Publishing Inc. This work is licensed under the Creative Commons Attribution International License (CC BY 4.0).

http://creativecommons.org/licenses/by/4.0/

\begin{abstract}
Introduction: Interdialytic weight-gain (IDWG) has been linked to various complications in hemodialysis (HD) patients. Method: Prospective clinical-observational study to evaluate the effect of IDWG in HD patients on the rate of hospital admissions over a 12-month period, and the impact of high IDWG on the frequency of IDH. Results: Of the 240 patients, those who had IDWG $\geq 4 \%, 81 \%$ had at least one hospital admission due to volume-overload or the need for extra HD-session(s). On the other hand, only $19 \%$ of those having IDWG $<4 \%$ had been admitted or got extra HD sessions $(\mathrm{p}<0.001)$. Of those who were admitted (over 12 months) due to volume overload; $74.1 \%$ had IDWG $\geq 4 \%$, while $25.9 \%$ had IDWG $<4 \%$ ( $p<0.001$ ). Regarding IDH, $87 \%$ of patients having IDWG $\geq 4 \%$ had at least one episode of IDH/week. On the other hand, only $22.5 \%$ of those with IDWG $<4 \%$ had one episode of IDH/week $(p<0.001)$. When analyzing those who had at least one IDH episode/week; $72.9 \%$ of them had IDWG $\geq 4 \%$, while only $27.1 \%$ had IDWG < $4 \%(\mathrm{p}<0.001)$. Conclusion: In HD patients, the frequency of hospital admission due to volume-overload and the need for extra HD-sessions is strongly related to the amount of IDWG ( $>4 \%$ in our patients), the same stands for the frequency of IDH. Thus, control of IDWG in HD patients is of great importance, keeping in mind the importance of the nutrition status of HD patients that may also impact IDWG.
\end{abstract}

\section{Keywords}

Interdialytic Weight-Gain, Hemodialysis, Hospital Admissions, Intradialytic Hypotension 


\section{Introduction}

The Gulf Cooperation Countries (GCC) has a relatively young population plagued with diabetes, hypertension, and obesity. The prevalence of dialysis in this region is high with diabetes being responsible for more than half of the cases. Hemodialysis is the preferred modality for most patients. It shows a low prevalence of blood-borne viruses (HBV, HCV, and HIV). Cancer rates in this region are low. Patients' reported quality of life in the GCC is comparable to other dialysis outcome practice pattern study (DOPPS) regions [1] [2] [3].

Interdialytic weight gain (IDWG) has been linked to various complications in hemodialysis (HD) patients, especially cardiovascular (CV) complications [4]. High ultrafiltration (UF) rate in relation to dialysis time has also a similar impact in various studies [5] [6]. Furthermore, high IDWG has been associated with all-cause and CV mortality [2] [7]. End-stage kidney disease (ESKD) is becoming a major concern to health systems as the cost is getting higher. In Oman, almost one percent of the population has severe kidney dysfunction [8]. There are $24 \mathrm{HD}$ centers in Oman that are working 24 hours a day to enable the provision of dialysis care to more than 2000 patients with ESKD in various geographical areas [8] [9] [10] [11].

Objective assessment of fluid overload is a predictor of mortality in patients having chronic kidney disease (CKD) stages 4 and 5 patients [12]. Therefore, dialysis institutes have adopted various approaches to reduce IDWG among HD patients. These include dietary sodium and fluid restriction, appropriate dialysis duration with suitable UF rate, and adjusting dialysate sodium concentration. The recommended IDWG among HD patients varies in different guidelines; for example, the European best practice guidelines (EBPG) recommends that IDWG should not exceed $4 \%-4.5 \%$ of the dry weight [13].

In our center, we have recorded many cases with high IDWG who suffered from various symptoms necessitating hospital admission due to systemic volume overload or, at least, having one or more extra hemodialysis session(s) in addition to the already established dialysis schedule. We have also noticed frequent episodes of intradialytic hypotension (IDH) in those having high IDWG.

In view of the above, we investigated the trends of IDWG in patients on regular HD in the Royal hospital-dialysis center in Muscat, and our aims of this study were to examine: 1) trends of IDWG in our patients in relation to different variables and comorbidities, e.g., age, hypertension, diabetes, CV disease, etc.; 2) associations of high IDWG with a frequency of hospitalization to systemic volume overload and the need for extra dialysis sessions; and 3) the impact of high IDWG on the frequency of IDH.

\section{Subjects and Methods}

\subsection{Study Design}

This was a 12-month, prospective, observational study that was conducted at the 
hemodialysis center-Royal hospital in Muscat. The study was carried out in accordance with the principles contained in the declaration of Helsinki and was approved by the local ethics committee of the ministry of health of the Sultanate of Oman. The primary end points of this study were to evaluate the effect of IDWG in HD patients on the rate of hospital admissions over a 12-month period, and the impact of high IDWG on the frequency of IDH. The secondary end points of the study were to evaluate the trends of IDWG in chronic HD patients in relation to different variables and comorbidities, and to evaluate the impact of various risk factors on the IDWG in HD patients.

The inclusion criteria:

- Patients on regular HD thrice weekly.

- Those who had been on regular dialysis for at least 3 consecutive months before enrollment.

- Dialysis session duration of 4 hours.

Exclusion criteria:

- Patients having HD schedules other than thrice weekly.

- Those who had been on HD for less than 3 months.

- Patients having HD for less than 4 hours per session.

The inclusion criteria included the following: patients on regular HD thrice weekly, for at least 3 consecutive months before enrollment. Those with a schedule other than thrice weekly were excluded and those on HD less than 3 months. There was no age restriction. Patients were recruited consecutively (over 3 successive sessions) by each investigator in the HD unit. Data were collected for the computer-based system "Alshifa 3 plus". Recruitment period had been opened for 12 months. Patients who were recruited in the study were recorded in a specific patient's $\log$ form to ensure privacy of their data all over the study. Oral informed consent was obtained from all subjects.

\subsection{Subjects}

The study included 240 patients with ESKD on regular HD. All patients had been receiving HD for at least 3 months. HD sessions were performed based on 4 hours thrice weekly. The data collected included; age, gender, duration on HD (in months), pre- and post-HD body weights (on 3 successive sessions), pre- and post-HD blood pressure (BP) measurements (on 3 successive sessions), the type of vascular access and the average blood flow rate, dialysis efficiency (based on average $\mathrm{Kt} / \mathrm{V}$ ), and the presence or absence of residual kidney function.

The presence of various comorbidities has been recorded including diabetes, hypertension, coronary artery disease (CAD), peripheral vascular disease (PVD), heart failure (HF), cerebrovascular disease (CVD), and other CV disorders. Also, we recorded any diagnosed neurological or pulmonary disorder. Laboratory data included serum creatinine, serum albumin, and hemoglobin level. The number of antihypertensive medications and the dose of diuretics were also recorded if regularly prescribed for any patient. 


\subsection{Assessment and Calculation of IDWG, Average Blood Pressure, and IDH}

The estimated IDWG was calculated based on the average between pre- and post-dialysis weights that were recorded on 3 consecutive dialysis sessions. The estimated IDWG was expressed in kilograms and as a percentage of the patients' dry weight. We have noticed more hospital admissions and complications for those having IDWG $4 \%$ to $4.5 \%$ of their dry weight. Also, the mean average IDWG among the study population was $2.9 \pm 1.7 \mathrm{~kg}$ that is equivalent to $4.1 \% \pm$ $1.9 \%$ (see below in the results). Therefore, we considered an estimated average IDWG of $4 \%$ as a cutoff value when evaluating IDWG in our patients. The following formula was used in the study:

IDWG $=\{$ Pre-dialysis weight - Post-dialysis weight (of the prior session) $\} \div$ Dry weight $\times 100$. The result is expressed as a percentage of the dry weight.

Similarly, the average blood pressures were calculated based on the average blood pressure readings pre- and post-dialysis on the same 3 consecutive sessions. We defined IDH as the drop of systolic blood pressure of $20 \mathrm{mmHg}$ or more during HD compared to the pre-dialysis blood pressure and/or systolic blood pressure that is less than $90 \mathrm{mmHg}$.

\subsection{Statistical Analysis}

Statistical analysis was done using IBM SPSS ${ }^{\circledR}$ Statistics version 22 (IBM ${ }^{\circledR}$ Corp., Armonk, NY, USA). Numerical data were expressed as mean and standard deviation or median and range as appropriate. Qualitative data were expressed as frequency and percentage. Pearson's Chi-square test or Fisher's exact test was used to examine the relation between qualitative variables. Comparison between two groups was done using either Student $\mathrm{t}$-test for normally distributed quantitative data or Mann-Whitney test (non-parametric t-test) for not normally distributed quantitative data. Spearman-rho method was used to test correlation between numerical variables. All tests were two-tailed. A p-value $<0.05$ was considered significant.

\section{Results}

The demographic and laboratory data are shown in Table 1 . The study included 240 HD patients; of them $65 \%$ were males while $35 \%$ were females. All of them had a dialysis schedule of 4-hours, thrice-weekly HD sessions. The majority of the patients (84.2\%) had an arteriovenous fistula (AVF) as a dialysis access, $4.2 \%$ had an arteriovenous graft (AVG), while $11.7 \%$ had HD via a tunneled venous catheter.

All the patients completed the study. There were no mortalities during the 12-month enrollment period of the study. The mean age of the patients was 50.4 \pm 15.0 years, with a median of 54.5 years. Comorbidities are shown in Table 2 .

The mean duration on HD was $84.5 \pm 64.3$ months, with a median of 66.6 months. The mean systolic and diastolic blood pressures were $151.4 \pm 20.1 \mathrm{mmHg}$ 
and $76.0 \pm 13.7 \mathrm{mmHg}$, respectively. Regarding dialysis adequacy, the mean $\mathrm{Kt} / \mathrm{V}$ was $1.4 \pm 0.2$, and the mean blood flow rate was $277.2 \pm 38.6 \mathrm{ml} / \mathrm{min}$. The mean average IDWG was $2.9 \pm 1.7 \mathrm{~kg}$ that is equivalent to $4.1 \pm 1.9 \%$ of the dry weight. We have recorded that $40.2 \%$ of the patients had at least 1 episode of IDH per week.

The mean number of episodes of IDH among all patients was $0.7 \pm 0.9$ episode/week. The mean number of IDH episodes among those of developed IDH was $1.5 \pm 0.7$ episode/week, with a median of 1.0 episode/week. Forty five percent of the patients needed one or more extra HD session or hospital admission due to volume overload/year. Almost half of the study population (49.2\%) had no residual function with anuria.

Table 1. Demographic and laboratory characteristics of the study population.

$\begin{array}{cc}\text { Parameter } & \text { Value } \\ \text { Age } & 50.4 \pm 15.0 \\ \text { Duration on hemodialysis (in months) } & 84.5 \pm 64.3 \\ \text { Mean inter-dialytic weight gain (in kg) } & 2.9 \pm 1.7 \\ \text { Mean inter-dialytic weight gain percentage } & 4.1 \pm 1.9 \\ \text { Intra-dialytic hypotensive episodes/week } & 0.7 \pm 0.9 \\ \text { Mean kt/V } & 1.4 \pm 0.2 \\ \text { Mean blood flow rate, } \mathrm{ml} / \text { minute } & 277.2 \pm 38.6 \\ \text { Hemoglobin, } \mathrm{g} / \mathrm{dL} & 2.2 \pm 1.2 \\ \text { Serum albumin, } \mathrm{g} / \mathrm{L} & 10.9 \pm 1.3 \\ \text { Serum creatinine, } \mu \mathrm{g} / \mathrm{dL} & 38.8 \pm 4.5\end{array}$

IDH is the drop of systolic blood pressure of $20 \mathrm{mmHg}$ or more during HD compared to the pre-dialysis blood pressure and/or a systolic blood pressure that is less than $90 \mathrm{mmHg}$.

Table 2. Frequency of various comorbidities among the study population.

\begin{tabular}{cc}
\hline Comorbidity & Prevalence in the study population \\
\hline Diabetes mellitus & $45.8 \%$ \\
Hypertension & $75.8 \%$ \\
Coronary artery disease & $22.5 \%$ \\
Congestive heart failure & $7.5 \%$ \\
Other cardiovascular diseases & $11.7 \%$ \\
Cerebrovascular disease & $5.0 \%$ \\
Peripheral vascular disease & $13.3 \%$ \\
Chronic pulmonary disorders & $9.2 \%$ \\
\hline
\end{tabular}


We have recorded that $50.0 \%$ of those younger than 55 years had IDWG $\geq$ $4 \%$. On the other hand, only $31.7 \%$ of those aged 55 years or more had IDWG $\geq$ $4 \%(\mathrm{p}=0.041)$. There was no significant gender difference regarding IDWG. Among those who had IDWG $\geq 4 \%, 81 \%$ of these patients had at least one hospital admission due to volume overload or the need for extra HD session(s). On the other hand, only $19 \%$ of those having IDWG $<4 \%$ had been admitted or got extra HD sessions $(\mathrm{p}<0.001)$. Of those who were admitted (over 12 months) due to volume overload (Figure 1); $74.1 \%$ had IDWG $\geq 4 \%$, while $25.9 \%$ had IDWG $<4 \%(\mathrm{p}<0.001)$.

Regarding IDH, $87 \%$ of patients having IDWG $\geq 4 \%$ had at least one episode of IDH/week. On the other hand, only $22.5 \%$ of those with IDWG $<4 \%$ had one episode of IDH/week $(\mathrm{p}<0.001)$. When analyzing those who had at least one IDH episode/week (Figure 2); $72.9 \%$ of them had IDWG $\geq 4 \%$, while only $27.1 \%$ had IDWG $<4 \%(\mathrm{p}<0.001)$. Other comorbidities (such as diabetes, hypertension, CV disease, and other disorders mentioned above) had no significant impact on the rate of hospital admission due to volume overload. Similarly, anemia and absence of residual kidney function had no statistically significant impact on admission rates.

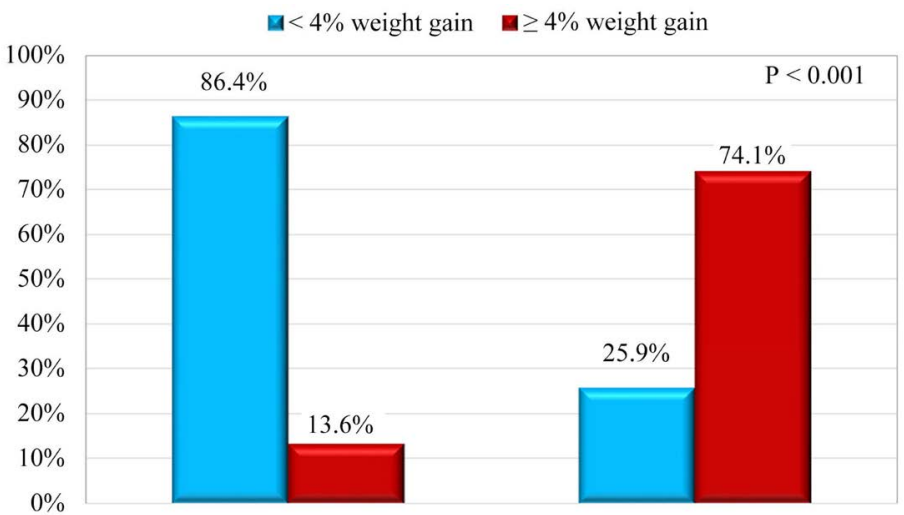

Figure 1. It shows the percentage of admitted patients due to volume overload classified according to Interdialytic weight-gain (IDWG).

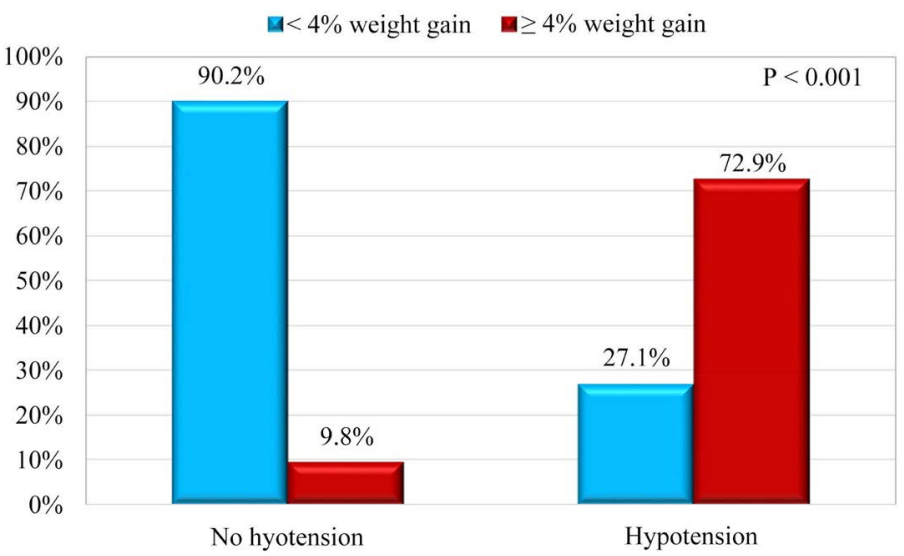

Figure 2. It shows the rate of intradialytic hypotension (IDH) among the study population related to Interdialytic weight-gain (IDWG). 


\section{Discussion}

This study evaluated the impact of IDWG on the rate of hospital admissions due to volume overload and the frequency of IDH. Studying the target IDWG is of great importance because excessive IDWG has been linked with unfavorable clinical consequences [14] [15], and is considered an independent predictor of all-cause and cardiovascular mortality in patients undergoing long-term HD [16]. In addition, IDWG is associated with increases in blood pressure and left ventricular hypertrophy; both of which can increase the risk of CV mortality in patients with ESKD. Furthermore, IDWG is an indicator of non-adherence to dialysis therapy, which is considered a significant predictor of mortality and hospitalization among HD population [17].

In most cases, ESKD patients are entirely dependent on the dialysis procedure for fluid removal. In fact, greater volumes of retained fluid increase cardiac filling pressures and predispose patients to left ventricular strain, pulmonary edema, and high blood pressure. In addition, because dialysis treatment time is essentially fixed for all patients, greater IDWG implies a greater rate of fluid removal during dialysis (i.e., ultrafiltration), which is associated with hemodynamic instability with increased morbidity and mortality [18].

Factors affecting IDWG significantly include the amount of oral fluid intake, dietary sodium, inadequate ultrafiltration (e.g., due to hypotension), diuretic use, and inappropriate dialysis prescription (e.g., unsuitable sodium profiling). It is worth noting that IDWG is related to the amount of food, fluid, and salt intake. Hence, researchers reported a significant positive correlation between IDWG and nutritional markers, which include the body mass index (BMI), normalized protein catabolic rate (nPCR), serum albumin, and creatinine [19] [20] [21] [22] [23]. This should be also considered during follow up of HD patients, as not all weight gain is only attributed to fluid intake. Dietary modification is one of the most important factors for controlling and reducing IDWG. Patients on regular HD should be instructed to limit oral fluid intake and sodium restriction. It is the duty of the dietitian and all staff members of the dialysis facility to continuously monitor the patients' nutritive status and fluid status. Not all chronic dialysis patients are compliant, and thus, repeated advice is recommended.

Furthermore, proper dialysis prescription has a direct impact on IDWG. This includes adequate UF, sodium profiling and dialysis time. The use of oral diuretics can be of value in patients on regular HD with residual urine output. Higher doses of loop diuretics are often needed (e.g., frusemide $80-120 \mathrm{mg}$ BID).

Many reports, such as that by Karmer et al., stated that high UF rates had been associated with increased mortality, and that limiting UF rates might be the way to improve the clinical outcomes [24]. They also stated that a policy establishing a UF rate limit could increase the risk for unintended consequences, including complications related to long-term volume overloads such as increased blood 
pressure and heart failure. They, in addition, advocated that probably for many patients, increasing HD session length or adding additional sessions will be required to avoid high UF rates, which may be an unpalatable prospect for many patients [24].

The health system in Oman is very good and it's free for all its citizens and was well appraised by the world health organization report [25] [26]. All citizens are provided with the necessary means to proper health care. However, in our patients, prescribing an additional dialysis session was refused by some patients, as this represented an extra burden for the patient and his family. Behavioral changes, including moderation of salt and fluid intake have shown to be an effective measure in this regard. This was also the recommendation by Kermer et al., and they considered this an effective preventive approach.

In view of a high prevalence of non-communicable disease and high early CKD population, there is an important need to consider comprehensive management strategies that involve the blockage of high renin-angiotensin-aldosterone and the use of mineral corticosteroid receptor blockers [27]. A study by Bomback et al. found a shift of the volume-aldosterone curve in the HD population suggesting that ESKD is a state of high volume and inappropriately high aldosterone [28]. The study concluded that the volume-aldosterone relationship has an important clinical significance, as dialysis patients may benefit from both volume reduction and mineralocorticoid receptor blockade [29].

Analysis of the "Frequent Hemodialysis Network Trials" concluded that reduction in volume and sodium overload in HD patients may beneficially reduce left ventricular mass, which has been previously increased due to fluid loading and hypertension [30].

Determining whether the association between IDWG and mortality varies by diseases, comorbidities (e.g. heart failure), or nutritional status as mirrored by various parameters (e.g. serum albumin). The HD staff's duty is to advise patients and their family members of their individual risk of IDWG based on the patients' conditions and aid in dietary counseling and control of other risk factors such as hypertension [31].

The target of ideal IDWG in HD patients varies among studies and various guidelines. For example, the international DOPPS study showed that IDWG > $5.7 \%$ versus $\leq 5.7 \%$ was associated with higher mortality [32]. Other studies in the USA showed that IDWG $>2.3 \%$ versus $\leq 2.3 \%$ and IDWG $>5.7 \%$ versus $\leq$ $5.7 \%$ were associated with higher mortality (HR 1.12 and 1.35, respectively) [33]. In the present study, IDWG ( $\geq 4 \%$ vs. $<4 \%$ ) was associated with no mortality among our patients over a one-year study period. Long-term follow-up is, thus, recommended for further evaluation. Variation in mortality rates can also be attributed to different demography, different dialysis practices, variable socio-economic status, various degrees of IDWG categories, and other comorbidities.

High IDWG is a potentially modifiable risk factor for adverse clinical outcomes and hospital admission. It is challenging for the patient, in most cases, to 
limit his/her oral fluid intake and control thirst sensation. We have advocated various approaches to deal with this including health education of patients, family and social support, and dietary counseling by a specialized dietitian. Certainly, a higher IDWG can serve as a positive marker of food intake, for the satisfaction of energy and protein demands, and this may be associated with increased oral fluid intake leading to a higher IDWG. Thus, the HD population should be continuously advised to limit the intake of salt and foods high in sodium, to enhance thirst control, favoring the lower fluid intake and IDWG [34].

Patients with ESKD on HD primarily have osmometric thirst due to oral salt intake. However, during HD, diffusive sodium transfer may occur. Just after the $\mathrm{HD}$, patients may also experience volumetric thirst due to hypovolemia because of UF and fluid depletion [35]. Both, dietary and dialytic sodium restriction can possibly hinder volume overload in the HD population according to some studies [36].

According to the European Best Practice Guidelines (EBPG), Fluid intake should not be necessarily adjusted for age, weight, gender, or body composition [13]. The guideline suggested 0.5 to 1 liter of oral fluid/day in addition to daily urine output, and that a $4.0 \%-4.5 \%$ weight gain as a percentage of the patient's dry weight would be acceptable in the majority of patients who follow the appropriate nutritional behavior and salt restriction [13]. In our study, $81 \%$ of patients having IDWG $\geq 4 \%$ had at least one hospital admission due to volume overload or the need for extra HD session(s). On the other hand, only $19 \%$ of those having IDWG $<4 \%$ had been admitted or got extra HD sessions ( $\mathrm{p}<$ 0.001).

Another important finding of this study is the significantly high rates of IDH episodes for those with high IDWG. We found that $87 \%$ of patients having IDWG $\geq 4 \%$ had at least one episode of IDH/week. On the other hand, only $22.5 \%$ of those with IDWG $<4 \%$ had one episode of IDH/week $(p<0.001)$ IDH has been known to be associated with increased cardiovascular and all-cause mortality [37] [38]. Patients having frequent IDH are eventually less tolerant to fluid removal by UF.

The HD procedure itself can cause reduced organ perfusion. An important example of this is the "myocardial stunning" which is transient, often significant, regional wall-motion abnormalities of the myocardium during HD and is known to be associated with cardiac systolic dysfunction and increased risk of death [39]. Low systolic blood pressure is a remarkable risk factor for myocardial stunning [40]. Studies have also shown that IDH has been linked to dementia (due to cerebral ischemia) and intestinal ischemia [41] [42].

The rate of UF has been linked to IDH. When the rate of fluid removal exceeds the rate of vascular space refilling from the interstitium, a decline in blood volume is evident, and IDH can develop [43]. One study showed that when the rate of UF exceeds $13 \mathrm{ml} / \mathrm{kg} /$ hour (which is equivalent to $910 \mathrm{ml} / \mathrm{hour}$ in a $70 \mathrm{~kg}$ adult) is associated with a high risk of developing IDH and higher mortality [44]. 
This is very clear in this study, where patients having high IDWG develop IDH much more frequently when having the same dialysis time despite having more fluid overload. This mandates higher rates of UF to achieve the target dry weight, with the risk of more frequent episodes of IDH.

Studies that evaluated the accuracy relative blood volume monitoring tool during $\mathrm{HD}$ as a guide for appropriate UF rate showed variable results but can be considered as an "add-on" parameter guiding UF in dialysis prescription [45]. Bioimpedance spectroscopy (BIS) has been used on a wider scale recently as a trial to reduce extracellular fluid depletion or overload, and to monitor fluid states during HD. There is some evidence that BIS-guided HD treatment may improve pre-dialytic fluid overload and hemodynamic tolerance. However, these studies are relatively small, and their results cannot be validated or used on a wide scale yet [46] [47]. Furthermore, in malnourished patients and in the elderly, the interpretation of BIS values is complicated [48]. More research is needed to evaluate its validity and applicability.

\section{Conclusions}

We have recorded significant hospital admissions (due to volume overload) and/or the need for additional HD sessions for patients with ESKD on regular HD who had IDWG of $\geq 4 \%$ of their dry weights. Similarly, these patients have significantly much more episodes of IDH which is related to the rate of UF. High IDWG has been linked to increase cardiac filling pressures and predispose patients to left ventricular strain, pulmonary edema, and high blood pressure. Frequent episodes of IDH are often associated with increased cardiovascular and all-cause mortality.

Therefore, we recommend that all healthcare providers in HD institutes should do their best for avoiding high IDWG including dietary advice and proper adjustments of HD patients' dry weights, and regular follow-up. Appropriate dialysis prescription should be individualized including the UF rate. The target should always be avoiding excessive IDWG due to fluid overload and avoiding malnutrition which may exert many adverse outcomes in HD patients. A perfect balance should always be the target.

\section{Disclosure of Potential Conflicts of Interest}

The study was approved by the Scientific Research Committee and certified that the study was performed in accordance with the ethical standards as laid down in the 1964 Declaration of Helsinki and its later amendments ethical standards. https://mohcsr.gov.om/my-researches/

\section{Consent for Publication}

All authors have agreed to the publication and to be accountable for all aspects of the work in ensuring that questions related to the accuracy or integrity of any part of the work are appropriately investigated and resolved. 


\section{Data Availability Statement}

Data can be provided upon request on individual basis but it is not available publicly.

\section{Author Contribution Statement}

All authors have contributed equally.

\section{Acknowledgements}

We would like to thank all our colleagues at the Research Ethics Committee and the Information Technology Department for their help.

\section{Conflicts of Interest}

The authors declare no conflicts of interest regarding the publication of this paper.

\section{References}

[1] AlSahow, A., AlRukhaimi, M., Al Wakeel, J., Al-Ghamdi, S.M., AlGhareeb, S., AlAli, F., Al Salmi, I., AlHelal, B., AlGhonaim, M., Bieber, B.A., Pisoni, R.L. and GCC-DOPPS 5 Study Group (2016) Demographics and Key Clinical Characteristics of Hemodialysis Patients from the Gulf Cooperation Council Countries Enrolled in the Dialysis Outcomes and Practice Patterns Study Phase 5 (2012-2015). Saudi Journal of Kidney Disease and Transplantation, 27, 12-23. https://doi.org/10.4103/1319-2442.194885

[2] Shaheen, F.A., Al Wakeel, J., Al-Ghamdi, S.M., Alhelal, B., AlGhareeb, S., Al Obaidli, A.A., AlSalmi, I., Abdulaziz, H.E., Bieber, B.A., Pisoni, R.L. and GCC-DOPPS 5 Study Group (2016) Cardiovascular and Cerebrovascular Comorbidities in Hemodialysis Patients from the Gulf Cooperation Council Countries Enrolled in the Dialysis Outcome and Practice Pattern Study Phase 5 (2012-2015). Saudi Journal of Kidney Disease and Transplantation, 27, S24-S30. https://doi.org/10.4103/1319-2442.194886

[3] Abouchacra, S., Obaidli, A., Al-Ghamdi, S.M., Al Wakeel, J., Al Salmi, I., Al Ghareeb, S., Al Azmi, M., Elsayed, M., Bieber, B.A., Pisoni, R.L. and GCC-DOPPS 5 Study Group (2016) Gulf Cooperation Council-Dialysis Outcomes and Practice Patterns Study: An Overview of Anemia Management Trends at the Regional and Country Specific Levels in the Gulf Cooperation Council Countries. Saudi Journal of Kidney Disease and Transplantation, 27, 51-61. https://doi.org/10.4103/1319-2442.194895

[4] Flythe, J.E., Kimmel, S.E. and Brunelli, S.M. (2011) Rapid Fluid Removal during Dialysis Is Associated with Cardiovascular Morbidity and Mortality. Kidney International, 79, 250-257. https://doi.org/10.1038/ki.2010.383

[5] Kalantar-Zadeh, K., Regidor, D.L., Kovesdy, C.P., et al. (2009) Fluid Retention Is Associated with Cardiovascular Mortality in Patients Undergoing Long-Term Hemodialysis. Circulation, 119, 671-679. https://doi.org/10.1161/CIRCULATIONAHA.108.807362

[6] Saran, R., Bragg-Gresham, J.L., Levin, N.W., et al. (2006) Longer Treatment Time and Slower Ultrafiltration in Hemodialysis: Associations with Reduced Mortality in the DOPPS. Kidney International, 69, 1222-1228. 
https://doi.org/10.1038/sj.ki.5000186

[7] Kimmel, P.L., Varela, M.P., Peterson, R.A., et al. (2000) Interdialytic Weight Gain and Survival in Hemodialysis Patients: Effects of Duration of ESRD and Diabetes Mellitus. Kidney International, 57, 1141-1151. https://doi.org/10.1046/j.1523-1755.2000.00941.x

[8] Al Majarfi, A., Al Salmi, A., Metry, A.M., Al Ismaili, F., Hola, A. and Hannawi, S. (2018) Epidemiology of Patients at Initial Treatment with Hemodialysis. ARC Journal of Nephrology, 3, 6-12.

[9] Al Ismaili, F., Al Salmi, I., Al Maimani, Y., et al. (2017) Epidemiological Transition of End-Stage Kidney Disease in Oman. Kidney International Reports, 2, 27-35. https://doi.org/10.1016/j.ekir.2016.09.001

[10] Al Alawi, I., Al Salmi, I., Al Mawali, A., Al Maimani, Y. and Sayer, J.A. (2017) End-Stage Kidney Failure in Oman: An Analysis of Registry Data with an Emphasis on Congenital and Inherited Renal Diseases. International Journal of Nephrology, 2017, Article ID: 6403985. https://doi.org/10.1155/2017/6403985

[11] Al Alawi, I.H., Al Salmi, I., Al Mawali, A. and Sayer, J.A. (2017) Kidney Disease in Oman: A View of the Current and Future Landscapes. Iranian Journal of Kidney Diseases, 11, 263-270. https://doi.org/10.1155/2017/6403985

[12] Liang, X., Wang, W. and Li, H. (2013) Water and Sodium Restriction on Cardiovascular Disease in Young Chronic Hemodialysis Patients. Chinese Medical Journal, 126, 1667-1672.

[13] Fouque, D., Vennegoor, M. and Ter Wee, P. (2007) EBPG Guideline on Nutrition. Nephrology Dialysis Transplantation, 22, ii45-ii87. https://doi.org/10.1093/ndt/gfm020

[14] Kim, D.Y., Kim, B., Moon, K.H., Lee, S. and Lee, D.Y. (2014) Effect of Gradually Lowering Dialysate Sodium Concentration on the Interdialytic Weight Gain, Blood Pressure, and Extracellular Water in Anuric Hemodialysis Patients. Renal Failure, 36, 23-27. https://doi.org/10.3109/0886022X.2013.830360

[15] Lee, M.J., Doh, F.M., Kim, C.H., Koo, H.M., Oh, H.J., Park, J.T., et al. (2014) Interdialytic Weight Gain and Cardiovascular Outcome in Incident Hemodialysis $\mathrm{Pa}$ tients. American Journal of Nephrology, 39, 427-435. https://doi.org/10.1159/000362743

[16] Movilli, E., Camerini, C., Gaggia, P., Zubani, R., Feller, P., Poiatti, P., et al. (2013) Magnitude of End-Dialysis Overweight Is Associated with All-Cause and Cardiovascular Mortality: A 3-Year Prospective Study. American Journal of Nephrology, 37, 370-377. https://doi.org/10.1159/000349931

[17] Nie, Y., Zhang, Z., Zou, J., Liang, Y., Cao, X., Liu, Z., et al. (2016) Hemodialysis-Induced Regional Left Ventricular Systolic Dysfunction. Hemodialysis International, 20, 564-572. https://doi.org/10.1111/hdi.12434

[18] Al Salmi, I., Larkina, M., Wang, M., Subramanian, L., Morgenstern, H., Jacobson, S., Hakim, R., Tentori, F., Saran, R., Akiba, T., Tomilina, N.A., Port, F.K., Robinson, B.M. and Pisoni, R.L. (2018) Missed Hemodialysis Treatments: International Variation, Predictors, and Outcomes in the Dialysis Outcomes and Practice Patterns Study. American Journal of Kidney Diseases, 72, 634-643. https://doi.org/10.1053/j.ajkd.2018.04.019

[19] Fischbach, M., Zaloszyc, A. and Shroff, R. (2015) The Interdialytic Weight Gain: A Simple Marker of Left Ventricular Hypertrophy in Children on Chronic Haemodialysis. Pediatric Nephrology, 30, 859-863.

https://doi.org/10.1007/s00467-015-3086-6 
[20] Garagarza, C.A., Valente, A.T., Oliveira, T.S. and Caetano, C.G. (2015) Effect of Personalized Nutritional Counseling in Maintenance Hemodialysis Patients. $\mathrm{He}$ modialysis International, 19, 412-418. https://doi.org/10.1111/hdi.12260

[21] Sakai, A., Hamada, H., Hara, K., Mori, K., Uchida, T., Mizuguchi, T., et al. (2017) Nutritional Counseling Regulates Interdialytic Weight Gain and Blood Pressure in Outpatients Receiving Maintenance Hemodialysis. The Journal of Medical Investigation, 64, 129-135. https://doi.org/10.2152/jmi.64.129

[22] Sezer, S., Kulah, E., Ozdemir, F.N., Tutal, E., Arat, Z. and Haberal, M. (2004) Clinical Consequences of Intermittent Elevation of C-Reactive Protein Levels in Hemodialysis Patients. Transplantation Proceedings, 36, 38-40. https://doi.org/10.1016/j.transproceed.2003.11.022

[23] Sezer, S., Ozdemir, F.N., Arat, Z., Perim, O., Turan, M. and Haberal, M. (2002) The Association of Interdialytic Weight Gain with Nutritional Parameters and Mortality Risk in Hemodialysis Patients. Renal Failure, 24, 37-48. https://doi.org/10.1081/JDI-120002659

[24] Kramer, H., Yee, J., Weiner, D.E., Bansal, V., Choi, M.J., Brereton, L., et al. (2016) Ultrafiltration Rate Thresholds in Maintenance Hemodialysis: An NKF-KDOQI Controversies Report. American Journal of Kidney Diseases, 68, 522-532. https://doi.org/10.1053/j.ajkd.2016.06.010

[25] Al Salmi, I. and Hannawi, S. (2016) The World Health Report-Health Systems Empowering Citizens and Improving Performance. Research on Humanities and Social Sciences, 6, 6.

[26] Al Salmi, I. and Hannawi, S. (2018) Health Workforce in the Sultanate of Oman: Improving Performance and the Health System. Journal of Internal Medicine and Patient Care Health, 1, 6.

[27] Osman, W., Al Dohani, H., Al Hinai, A.S., et al. (2020) Aldosterone Renin Ratio and Chronic Kidney Disease. Saudi Journal of Kidney Disease and Transplantation, 31, 70-78. https://doi.org/10.4103/1319-2442.279963

[28] Bomback, A.S., Kshirsagar, A.V., Ferris, M.E. and Klemmer, P.J. (2009) Disordered Aldosterone-Volume Relationship in End-Stage Kidney Disease. Journal of the Renin-Angiotensin-Aldosterone System: JRAAS, 10, 230-236. https://doi.org/10.1177/1470320309352353

[29] Raimann, J.G., Chan, C.T., Daugirdas, J.T., Depner, T., Gotch, F.A., Greene, T., et al. (2016) The Effect of Increased Frequency of Hemodialysis on Volume-Related Outcomes: A Secondary Analysis of the Frequent Hemodialysis Network Trials. Blood Purification, 41, 277-286. https://doi.org/10.1159/000441966

[30] Kim, J.C., Kalantar-Zadeh, K. and Kopple, J.D. (2013) Frailty and Protein-Energy Wasting in Elderly Patients with End Stage Kidney Disease. Journal of the American Society of Nephrology, 24, 337-351. https://doi.org/10.1681/ASN.2012010047

[31] Wong, M.M., McCullough, K.P., Bieber, B.A., Bommer, J., Hecking, M., Levin, N.W., et al. (2017) Interdialytic Weight Gain: Trends, Predictors, and Associated Outcomes in the International Dialysis Outcomes and Practice Patterns Study (DOPPS). American Journal of Kidney Diseases, 69, 367-379. https://doi.org/10.1053/j.ajkd.2016.08.030

[32] Hecking, M., Moissl, U., Genser, B., Rayner, H., Dasgupta, I., Stuard, S., et al. (2018) Greater Fluid Overload and Lower Interdialytic Weight Gain Are Independently Associated with Mortality in a Large International Hemodialysis Population. Nephrology Dialysis Transplantation, 33, 1832-1842.

https://doi.org/10.1093/ndt/gfy083 
[33] Sherman, R.A., Cody, R.P., Rogers, M.E. and Solanchick, J.C. (1995) Interdialytic Weight Gain and Nutritional Parameters in Chronic Hemodialysis Patients. American Journal of Kidney Diseases, 25, 579-583. https://doi.org/10.1016/0272-6386(95)90126-4

[34] Lindley, E.J. (2009) Reducing Sodium Intake in Hemodialysis Patients. Seminars in Dialysis, 22, 260-263. https://doi.org/10.1111/j.1525-139X.2009.00570.x

[35] Kooman, J.P., van der Sande, F., Leunissen, K. and Locatelli, F. (2003) Sodium Balance in Hemodialysis Therapy. Seminars in Dialysis, 16, 351-355. https://doi.org/10.1046/j.1525-139X.2003.16070.x

[36] Hecking, M., Karaboyas, A., Saran, R., Sen, A., Inaba, M., Rayner, H., Horl, W.H., Pisoni, R.L., Robinson, B.M., Sunder-Plassmann, G. and Port, F.K. (2012) Dialysate Sodium Concentration and the Association with Interdialytic Weight Gain, Hospitalization, and Mortality. Clinical Journal of the American Society of Nephrology, 7, 92-100. https://doi.org/10.2215/CJN.05440611

[37] Flythe, J.E., Xue, H., Lynch, K.E., Curhan, G.C. and Brunelli, S.M. (2015) Association of Mortality Risk with Various Definitions of Intradialytic Hypotension. Journal of the American Society of Nephrology, 26, 724-734.

https://doi.org/10.1681/ASN.2014020222

[38] Stefánsson, B.V., Brunelli, S.M., Cabrera, C., Rosenbaum, D., Anum, E., Ramakrishnan, K., et al. (2014) Intradialytic Hypotension and Risk of Cardiovascular Disease. Clinical Journal of the American Society of Nephrology, 9, 2124-2132. https://doi.org/10.2215/CJN.02680314

[39] Assa, S., Hummel, Y.M., Voors, A.A., Kuipers, J., Westerhuis, R., de Jong, P.E., et al. (2012) Hemodialysis-Induced Regional Left Ventricular Systolic Dysfunction: Prevalence, Patient and Dialysis Treatment-Related Factors, and Prognostic Significance. Clinical Journal of the American Society of Nephrology, 7, 1615-1623. https://doi.org/10.2215/CJN.00850112

[40] Burton, J.O., Jefferies, H.J., Selby, N.M. and McIntyre, C.W. (2009) Hemodialysis-Induced Cardiac Injury: Determinants and Associated Outcomes. Clinical Journal of the American Society of Nephrology, 4, 914-920. https://doi.org/10.2215/CJN.03900808

[41] Assimon, M.M., Wang, L. and Flythe, J.E. (2019) Cumulative Exposure to Frequent Intradialytic Hypotension Associates with New-Onset Dementia among Elderly Hemodialysis Patients. Kidney International Reports, 4, 603-606.

https://doi.org/10.1016/j.ekir.2019.01.001

[42] Seong, E.Y., Zheng, Y., Winkelmayer, W.C., Montez-Rath, M.E. and Chang, T.I. (2018) The Relationship between Intradialytic Hypotension and Hospitalized Mesenteric Ischemia: A Case-Control Study. Clinical Journal of the American Society of Nephrology, 13, 1517-1525. https://doi.org/10.2215/CJN.13891217

[43] Mitsides, N., Pietribiasi, M., Waniewski, J., Brenchley, P. and Mitra, S. (2019) Transcapillary Refilling Rate and Its Determinants during Haemodialysis with Standard and High Ultrafiltration Rates. American Journal of Nephrology, 50, 133-143. https://doi.org/10.1159/000501407

[44] Aronoff, G.R. (2017) The Effect of Treatment Time, Dialysis Frequency, and Ultrafiltration Rate on Intradialytic Hypotension. Seminars in Dialysis, 30, 489-491. https://doi.org/10.1111/sdi.12625

[45] Keane, D.F., Baxter, P., Lindley, E., Rhodes, L. and Pavitt, S. (2018) Time to Reconsider the Role of Relative Blood Volume Monitoring for Fluid Management in $\mathrm{He}$ modialysis. ASAIO Journal, 64, 812-818. 
https://doi.org/10.1097/MAT.0000000000000795

[46] Machek, P., Jirka, T., Moissl, U., Chamney, P. and Wabel, P. (2010) Guided Optimization of Fluid Status in Haemodialysis Patients. Nephrology Dialysis Transplantation, 25, 538-544. https://doi.org/10.1093/ndt/gfp487

[47] Chen, H.-S., et al. (2016) Application of Bioimpedance Spectroscopy in Asian Dialysis Patients (ABISAD-III): A Randomized Controlled Trial for Clinical Outcomes. International Urology and Nephrology, 48, 1897-1909. https://doi.org/10.1007/s11255-016-1415-8

[48] Keane, D.F., Bowra, K., Kearney, K. and Lindley, E. (2017) Use of the Body Composition Monitor for Fluid Status Measurements in Elderly Malnourished Subjects. ASAIO Journal, 63, 507-511. https://doi.org/10.1097/MAT.0000000000000508 